to keep the temperature between $160^{\circ}$ and $165^{\circ} \mathrm{C}$. in every individual oven.

I hope that this addition to the absolutely sure French dry air sterilization may contribute to a well-merited further use of this method, both by general surgeons and by eye operators ; especially for the latter, as the sharp point and edge are of the highest importance.

I wish to acknowledge my indebtedness to Dr. Th. Thjötta, who has undertaken all bacteriological controls mentioned in this paper and also called my attention to the automatic thermo-regulation of Heraeus, which caused me to attach this to the electrically heated dry air sterilizor of Lautenschläger.

The agent in England for W. C. Heraeus, Hanau a. Main, is : The Scientific Glass-Blowing Company, 12/14, Wright Street, Oxford Road, Manchester.

\title{
THE IDENTITY OF CATARACT FORMATIONS
}

BY

J. Foster, F.R.C.S.

LEEDS

THERE are still ophthalmologists in this country who prefer to use a loupe when a slit-lamp is available; possibly because they consider a loupe can reveal anything worth seeing, or possibly because of the large amount of confusing details the slit-lamp brings to light. A well used loupe may compete clinically, to a certain extent, with the slit-lamp so far as the anterior chamber is concerned, but for the lens the latter is incontestably superior. In this paper I have, therefore, made an attempt by pruning detail, and stressing practical points, to give a working classification of the types of cataract, and their appearance, as seen with the slitlamp. Nothing new is offered, and almost all the facts can be found in greater detail and different arrangement in Goulden's translation of Koby's "Slit-lamp Microscopy of the Living Eye."

Since the cause of a cataract is frequently a matter of the utmost interest from the prognostic, operative, or medico-legal aspect, the analysis of an otherwise amorphous lens opacity by the slitlamp beam is often a source of great satisfaction to the observer.

As the slit-lamp beam shines through the lens, attention must be paid, not only to the shape of the opacities, but also to the so-called "bands," and the zones of specular reflection. The former stand out as bright lines in the beam (drawn dark in Fig. 1) where areas of different refractive index, formed at different life stages, are in contact, and two Y-shaped figures are our marking 
for the limit of the foetal nucleus, though there may be seen outside this a less well marked peripheral band which shows the outer limit. The zones of specular reflection are the areas where light is reflected from the anterior and posterior surfaces of the lens as from a mirror. They are inspected for the presence or absence of polychromatic lustre. This, when present, looks like a shiny area of a map of England with different coloured counties side by side. An illustration better than any description, is obtained by inspecting the area of reflection from the posterior lens capsule of an aphakic eye prior to capsulotomy.

These optical phenomena are an additional help in identifying the age and cause of any cataract.

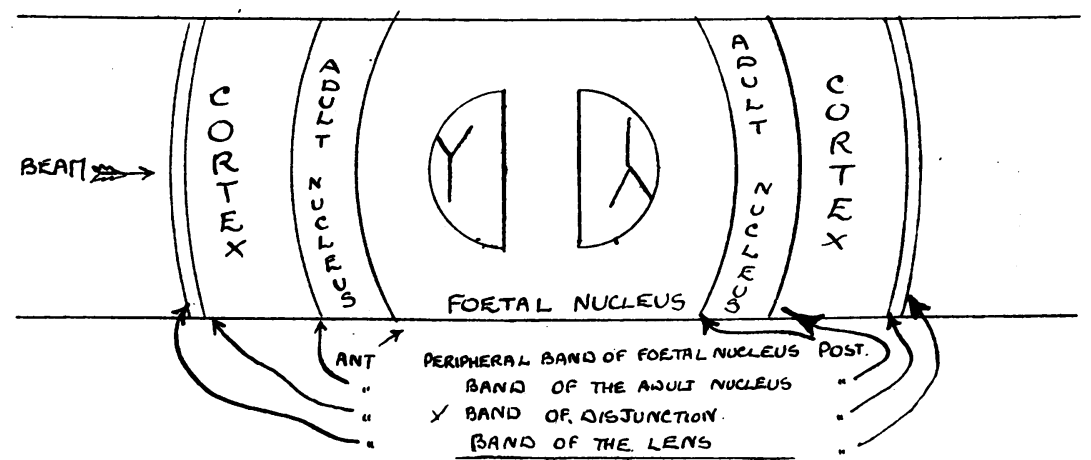

FIG. 1. Based on the conventional adult lens of Vogt.

\section{Classification}

(a) Congenital 1. "Kalk" type Anterior or posterior polar.

2. Punctate type.

(b) ACQUIRED

1. Presenile

Coronary.

"Water-spaces."

2. Senile

Nuclear.

Cuneiform.

Cupuliform.

Dust-like in concentric layers.

3. Complicated Uveal or retinal disease.

4. Caloric "Heat-workers."

5. Traumatic Indirect and direct violence.

6. Glandular Dyscrazia

Subcapsular-Diabetes.

Cortical-other diseases. 
(a) Congenital Cataracts. Are roughly divisible into two types. (1) The anterior and posterior polar, which are solid, chalk-white opacities, usually axial in situation. Occasionally, these exhibit a collar-stud shape due to re-duplication produced by the growth of the lens. Where the anterior polar opacities are very superficial, the cause is probably foetal iritis; this is borne out by strands running, in some cases, from the iris collarette to the opacity. The posterior polar opacities are usually due to hyaloid vascular anomalies. (2) The punctate variety. These tiny, regular spaced white dots (Fig. 2), are the units into which the pattern of a lamellar or other central congenital cataract resolves when subjected to a magnification of $\times 23$. If truly congenital, they are

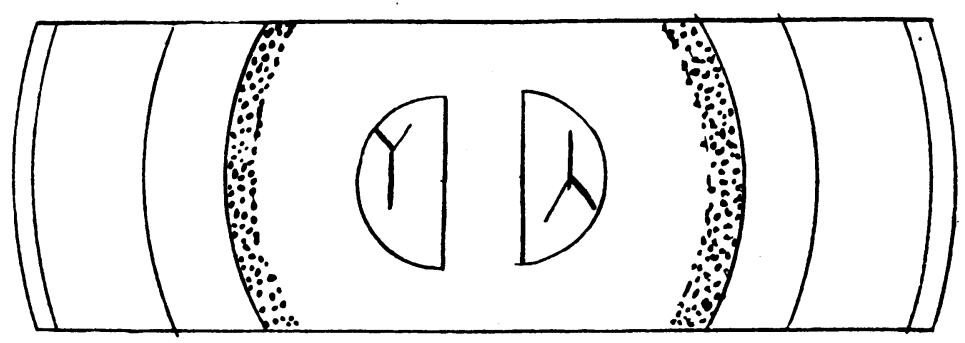

FIG. 2.

limited to the foetal nucleus and are usually concentrated at the zone marking its surface. Part of some lamellar cataracts arise after birth and are outside this limit. There are, of course, in addition, many other forms of congenital cataract differing in shape and arrangement from the above, but the majority are variants on these themes. Those given are the most important clinically, because the commonest.

(b) Acquired Cataracts. Senile and pre-senile opacities can be considered together as they frequently co-exist. Nuclear cataract is probably the commonest of the senile varieties over, and cuneiform under 65 years of age, but no one has yet worked out the relative incidence. Nuclear and cuneiform or cupuliform cataracts frequently co-exist.

In all adults over 60 years of age the lens between the bands of the adult nucleus becomes more yellowly luminous in the slit-lamp beam. This process is distinguished from nuclear cataract only in the matter of degree. Cuneiform cataract presents a familiar picture with the ophthalmoscope mirror of black spikes "aimed" at the axis of the lens. These opacities are in the cortex superficial to the adult nucleus. Water clefts, which appear at about 50 years of age, are of the same shape and situation, but can 
be seen to be transparent, with ophthalmoscope mirror and slit-lamp.

Coronary opacities are round, ring, or club-shaped, but the apices of the clubs are directed away from the axis of the lens. The opacities may be brown or blue in colour (blue-dot cataract), and are situated mainly in the periphery of the adult nucleus and, to a lesser extent, in the deep cortex (Fig. 3). They occur in about 30 per cent. of all patients. "Dust-like" cataract when it occurs, is usually associated with this, and is distinguished by extending into the pupillary area in the same layers. Pre-senile changes, as a rule, interfere much more slowly with vision than senile changes.

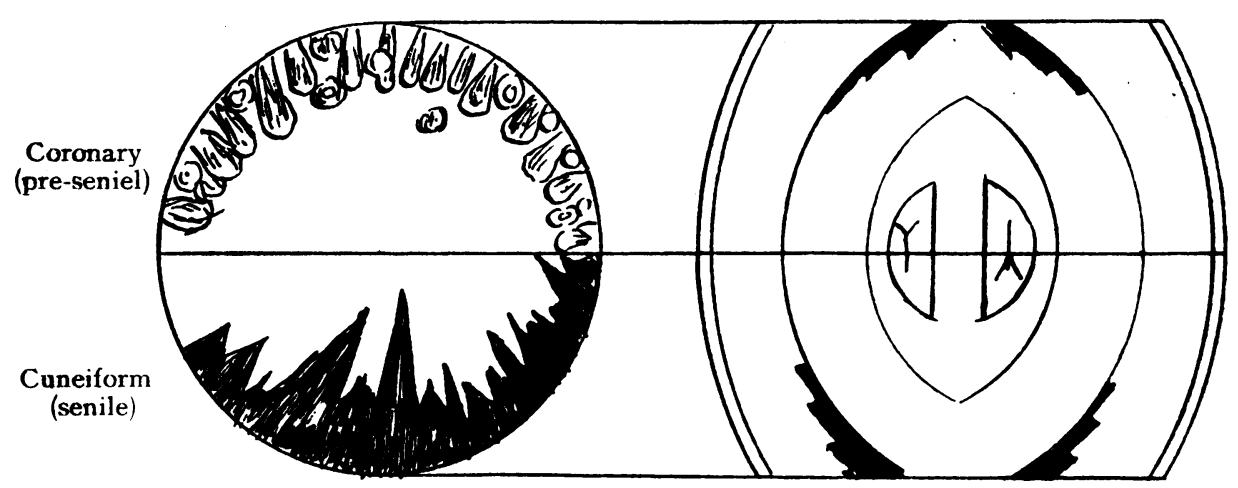

FIG. 3. After Goulden.

The discrimination of the last senile type, cupuliform or saucershaped cataract, is a triumph for the slit-lamp, as this is usually the sole means of distinguishing it from other posterior cortical opacities. The cataract, which is usually accompanied by equatorial cuneiform opacities, consists of a dome-shaped area of uniform thickness in the posterior cortex near the capsule. This dome extends to the periphery, but not axially. It is composed of dense, crystalline opacities of no great size, interspersed with rounded vacuoles. As there is no overlying haze, details are clear cut, and a golden lustre, which is even slightly polychromatic, may be seen. It is easy to distinguish it from complicated cataract by considering the above features unless a nuclear cataract is present in addition, in which case it may be very difficult.

(3) Complicated cataract is a progressive condition, and is difficult to describe shortly. The slit-lamp or ophthalmoscope may reveal in the early stages, as the causative condition, retinitis pigmentosa or iridocyclitis. A certain number appear to occur in otherwise normal eyes. It is possible, however, that such cases are really senile cupuliform cataracts with exaggerated lustre. 
A polychromatic lustre at the posterior zone of specular reflection is the earliest sign of the condition. Faint flaky opacities rendered indefinite by overlying haze, appear in the posterior cortex at the posterior pole gradually taking "rosette" form. The surface of this becomes bread-crumb-like or tufted, and extends forward axially. The lustre becomes obliterated except at the edges which extend slightly outwards. A few coloured crystals and vacuoles are now seen as the opacity advances, and a second opacity appears behind the posterior band of the adult nucleus. The opacity extends gradually until it is complete; shortly before which, it may be distinguished from a complete senile cataract by a polychromatic lustre from the anterior zone of specular reflection.

(4) Infra-red ray cataract is another form of posterior cortical cataract the slit-lamp has elucidated. One type is common to all "heat-workers," iron, tin-plate, or glass. Stoewers found in 2,000 glass-blowers that it hardly ever occurred before the 20th year of employment.

It is not surprising, therefore, that some confusion seems to have existed as to the form heat cataract might take. Robinson's original paper and those of some subsequent writers exemplify this, as forms are included which are probably senile. The characteristic opacity is disc-shaped and situated entirely in the axial and posterior layers of the lens. It is made up of small, flaky or speckled opacities which may glitter. Vacuoles are extremely rare. To the ophthalmoscope the cataractous disc appears irregular and porous, and may be sharply limited towards the periphery. There may even be a circular dark line around it. This is due to the protection of the iris-diaphragm which limits the area of lens exposed to infra-red rays to that of the pupil. The disc spreads forward axially to the centre of the lens. Associated with the earlier stages may be an unduly early diminution of accommodation. Apart from a rupture of the zonular layer of the anterior lens capsule found in 9 out of 32 cases there is nothing specially characteristic about the later stages of the condition. Vogt's experiment showing that ultra-violet sensitive photographic paper is unaffected by rays from a glass furnace at $2,000^{\circ} \mathrm{C}$. has ruled out these rays as the causative factor of this lesion.

(5) Traumatic cataracts are of interest in that they may become absorbed in part or completely. The opacity peculiar to trauma is distinctive. It consists of a "rosette," a thin white layer in the posterior cortex, looking like several white crossed feathers, the shafts of which (see Fig. 4) are the lens sutures accentuated by droplets formed between the fibres. The reflex is dead white, without polychromatic or other lustre. When caused by an intraocular foreign body, the capsular rent and slightly opaque track may be seen. Where the rent is large, white broken-up fibres and 


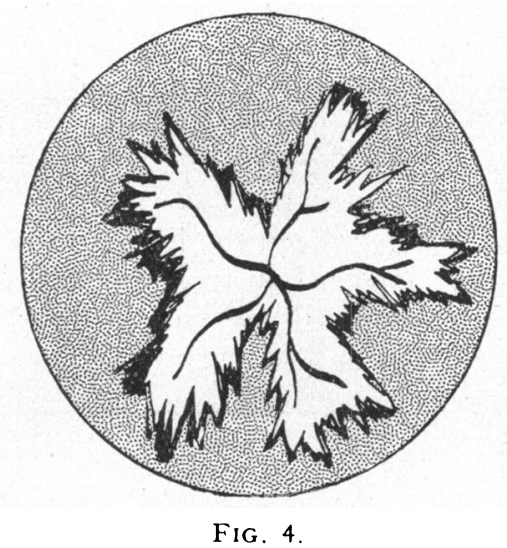

an area of accentuation of the fibre pattern may intervene between it and the remaining clear lens.

When due to indirect violence the "rosette" may be anterior, or posterior, or both, connected in some cases by flat angular opacities running axially through the intervening lens. It is this type due to indirect violence, described originally by Hudson, that sometimes disappears in such an extraordinary manner. When the injury has occurred during youth, the opacity may be buried as age advances, by fresh clear lens. Old opacities are stated to have the sutures, i.e., the shaft of the feather in the centre of the feather (division of rosette). The medico-legal importance of the fact that trauma produces a characteristic lesion in the lens can easily be judged if one imagines oneself faced with an old man with senile lens opacities, who, knocked down by a car, while recovering discovers his vision is not what it was and attributes this to a cataract from the blow.

If a traumatic cataract is complete it may yet be possible to detect signs of trauma to the iris or siderosis bulbi, and thereby determine its origin.

"Electric shock" cataract resembles the traumatic variety morphologically, though there is, in addition, a type with a central, anterior, sub-capsular droplet opacity.

(6) Cataracts due to glandular dyscrasia are of considerable interest to the physician as in some diseases of. dystrophia myotonica, in which they may be the first sign of the disease.

There are two types, the sub-capsular type due to diabetes is distinct from the others. It occurs in young people, aged 20-40 years, who are usually under treatment with insulin, and consists of small cloudy opacities situated anteriorly and posteriorly directly under the lens epithelium, between it and the zone of disjunction. These opacities may resemble droplets, or be grouped in areas 
of $0.1 \mathrm{~mm}$. in size. In addition, small coloured corpuscles and clear clefts separating the fibres may be seen. A case of rather similar opacities shown at the Royal Society of Medicine in a healthy young acetone worker raises a point of interest in connection with the aetiology (see Fig. 5).

The cortical type is caused by several diseases. The opacities which are situated more deeply in the lens (in the cortex) are small, flaky, or rounded, but coarser than in diabetes, and are interspersed with many coloured crystals. They vary a little in size and shape

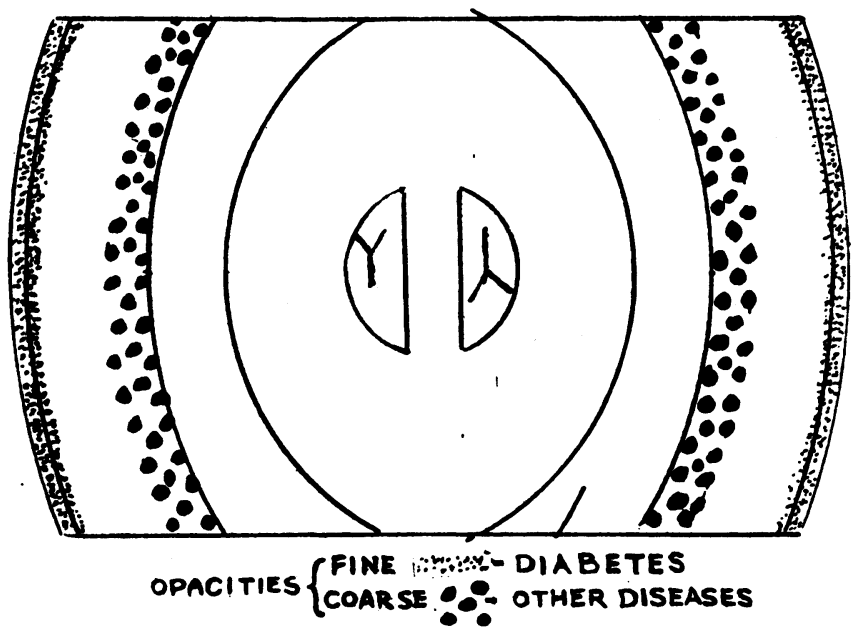

Fig. 5.

according to the disease. In dystrophia myotonica the opacities appear between 20 and 30 years of age, and are small and angular. In cretins, they are small and flaky, in both they are in the superficial layers of the cortex anteriorly and posteriorly. In operative myxoedema, the opacities are flattened, disc-like, bluish and as coarse as in coronary cataract. In mongolian idiots, about 9 years of age, punctate, flaky, and coloured opacities develop in all layers except immediately under the lens capsule and in the embryonic nucleus. Sometimes the opacities are arranged in a disc. Some months to a year after removal of the para-thyroids and the development of post-operative tetany, faint powdery crystalline opacities develop in the cortex of the lens, and tend to arrange themselves in a stellate form in the posterior cortex in some cases. As this type of cataract may also develop in cases of idiopathic tetany where the signs of the causative disease are not prominent, care must be taken in this diagnosis, as a spontaneous hyphaema is liable to occur on the fifth day after extraction. 